Conclusions Based on our initial data, we conclude that the Trevo NXT is an effective and safe tool for mechanical thrombectomy especially when used for combined approaches. Disclosure Nothing to disclose

\section{EP53* THE NOVEL TENZING INSERTS - THE SEAMLESS WAY TO REACH THE CLOT IN LARGE AND MIDDLE VESSEL CEREBRAL OCCLUSIONS - PRELIMINARY EXPERIENCE}

F Massari, J Singh, AL Kuhn, V Naragum, V Anagnostakou, MJ Gounis, AS Puri. University of Massachusetts, Worcester, MA, USA

\subsection{6/neurintsurg-2021-ESMINT.52}

Introduction-Objectives To report the feasibility, safety and efficacy in utilizing the novel Tenzing inserts during stroke thrombectomy in order to reach the clot with large bore catheters, without need for crossing.

Aims-Methods The distal trackability of the new generation of super large bore aspiration catheters is provided by the newly engineered Tenzing inserts, which present virtually atraumatic self-centering soft tapered tip, capable to navigate the tortuous cervico-cranial vasculature with very unfrequently necessity of an inner microwire, the extremely large shaft which almost zeroes the step-off with the aspiration catheters, reducing the risk to get stuck at the level of vascular ledges/bifurcations and the unnecessity to cross the embolus, which significantly reduce the risk of downstream clot embolization. The Tenzing 7 is compatible with large bore aspiration catheters, while the Tenzing 8 matches the 088' HiPoint super large bore catheter, which in combination with the Base Camp guiding catheter is utilized for the Mount Everest Technique (MET).

Results We have utilized this novel insert in 20 patients with large and moderate vessel occlusions involving the anterior (19; 8 ICA, 8 MCA-M1, 3 MCA-M2) and posterior ${ }^{1}$ circulation with $100 \%$ efficacy. No embolization in unaffected vascular territories or symptomatic SAH has been observed.

Conclusions In our preliminary experience, the utilization of the novel Tenzing inserts proved to be extremely effective in reaching the clot with large and super large bore catheters, without the necessity to cross the embolus as well as without encountering the issue of getting stuck at the level of vascular ledges/bifurcations.

\section{REFERENCE}

1. Frölich AM, Kim W, Stribrny K, Jansen O, Möhlenbruch M, Szikora I, Wodarg F, Fiehler J, Otto K, Chou T, Buhk JH, English J. The novel Tenzing 7 delivery catheter designed to deliver intermediate catheters to the face of embolus without crossing: clinical performance predicted in anatomically challenging model. J Neurointerv Surg 2020 Sep 3:neurintsurg-2020-016412.

Disclosure Nothing to disclose

\section{EP54* UTILITY OF DRUG-ELUTING COROFLEX ISAR STENT IN INTRACRANIAL ATHEROSCLEROTIC DISEASE: A SINGLE CENTER EXPERIENCE IN 147 PATIENTS}

A El Mekabaty, V Hellstern, M Auguilar-Perez, H Henkes. Diagnostic and Interventional Neuroradiology, Klinikum Stuttgart Katharienenhospital, Stuttgart, Germany

10.1136/neurintsurg-2021-ESMINT.53

Introduction Intracranial atherosclerotic disease (ICAD) is a major cause of stroke worldwide. Different treatment regimen, including medical treatment (e.g. dual antiplatelet inhibition) and endovascular treatment are available. We aim to investigate the safety and efficacy of the drug-eluting, balloonmounted Coroflex ISAR stent in patients with ICAD.

Methods A retrospective analysis of patients with ICAD and attempted implantation of Coroflex ISAR stent in our institution from 2014 to 2020 was performed.

Results A total of 147 patients were included (74.1\% males, average age 70 years 'range 47-93'). Median baseline modified Rankin score (mRS) was 0 (interquartile range 'IQR' $0-1$ ) and median pretreatment mRS was 2 (IQR 1-4). Lesions treated were located in the ICA (42.9\%), V4 segment (36.1\%), basilar artery (12.2\%) and M1 segment (8.8\%). Stent was successfully implanted in $92.6 \%(137 / 147)$ and the vessel stenosis was reduced from a mean of $76 \%$ 'range $50-99$ ' to $36 \%$ 'range 0-82'. Short-term follow-up was available in $77.7 \%$ of patients after a median of 3 months 'IQR 1-5' and long-term follow-up was available in 63.3\% (93/147) after a median of 24 months 'IQR 14-40'. In-Stent stenosis occurred in $13.9 \%$ $(16 / 115)$, recurrent stroke in $9.5 \%(11 / 115)$ and asymptomatic intracranial hemorrhage in $3.4 \%$ (5/147). Over-all mortality was $4.1 \%(6 / 147)$.

Conclusion The drug-eluting, balloon-mounted Coroflex ISAR is a safe and effective treatment option for ICAD. The overall recurrent stroke rate was $9.5 \%$ and mortality was $4.1 \%$. Further work is needed to better identifiy ICAD patients for stenting.

\section{REFERENCES}

1. Ye G, et al. Efficacy and safety of drug-eluting stent for the intracranial atherosclerotic disease: a systematic review and meta-analysis. J Clin Neurosci 2019 Jan;59:112-118

2. Vajda $Z$, et al. Treatment of intracranial atherosclerotic disease with a balloonexpandable paclitaxel eluting stent: procedural safety, efficacy and mid-term patency. Clin Neuroradiol 2012 Sep;22(3):227-33.

Disclosure Nothing to disclose

\section{EP55 KEEP IT SIMPLE: THE SNACE (SOFIA NON-WIRE ASPIRATION CONTRAST ENHANCEMENT) TECHNIQUE FOR THE ENDOVASCULAR MANAGEMENT OF ACUTE ISCHEMIC STROKE}

B Pabon, V Torres, J Mejia, M Patiño, 0 Vargas, N Serna, J Pelaez. Angioteam, Medellin, Colombia

\subsection{6/neurintsurg-2021-ESMINT.54}

Background The availability of advanced large-bore diameter aspiration catheters has improved recanalization rates and time. We report a prospectively collected clinical experience with a simple technique: SNACE (Sofia Non-wire Aspiration Contrast Enhancement) as the primary method for vessel recanalization.

Methods 38 prospective patients with ELVO at four institutions were included in the study. The SNACE technique was utilized in all patients. Procedural and clinical data were analyzed.

Results The SNACE approach using SOFIA 6 Plus Catheter was successful in achieving Thrombolysis in Cerebral Infarction (TICI) $2 \mathrm{~b}$ or 3 revascularizations in $88 \%$ of cases. The first Pass effect was obtained in $75 \%$. The average time from groin puncture to at least TICI $2 \mathrm{~b}$ recanalization was $17 \mathrm{~min}$. National Institutes of Health Stroke Scale (NIHSS) score average at the onset of 16 and improved to a median NIHSS 
score at discharge of 5.5. We did not find intraprocedural complications and two symptomatic intracerebral hemorrhages were recorded postoperatively.

Discussion The SNACE technique is a simple, fast, safe, and effective method that has reduced the requirements to multiple passes and avoiding the use of expensive materials to reach the occlusion site. SNACE is a replicable approach without additional training requirements.

\section{REFERENCES}

1. Gory B, Armoiry X, Sivan-Hoffmann R, Piotin M, Mazighi M, Lapergue B, et al. A direct aspiration first pass technique for acute stroke therapy: a systematic review and meta-analysis. Eur J Neurol 2018 Feb 1;25(2):284-92.

2. Blanc R, Redjem H, Ciccio G, Smajda S, Desilles JP, Orng E, et al. Predictors of the aspiration component success of a direct aspiration first pass technique (ADAPT) for the endovascular treatment of stroke reperfusion strategy in anterior circulation acute stroke. Stroke 2017 Jun 1;48(6):1588-93.

Disclosure Boris pabon proctorship con MEDTRONIC, Microvention Consultant MIVI

\section{EP56 TARGETED SINGLE LOW-DOSE INTRA-ARTERIAL BEVACIZUMAB FOR STEROID-REFRACTORY RADIATION NECROSIS OF THE BRAIN}

${ }^{1} \mathrm{SR}$ Dashti, ${ }^{2} \mathrm{RJ}$ Kadner, ${ }^{3} \mathrm{BS}$ Folley, ${ }^{4} \mathrm{JP}$ Sheehan, ${ }^{5} \mathrm{DY}$ Han, ${ }^{6} \mathrm{RJ}$ Kryscio, ${ }^{7} \mathrm{MB}$ Carter, ${ }^{3}$ LBE Shields, ${ }^{8} \mathrm{BM}$ Plato, ${ }^{9} \mathrm{RV}$ La Rocca, ${ }^{10} \mathrm{AC}$ Spalding, ${ }^{1} \mathrm{TL}$ Yao, ${ }^{11} \mathrm{JF}$ Fraser. ${ }^{1}$ Cerebrovascular and Endovascular Neurosurgery Institute, Norton Neuroscience Institute, Norton Healthcare; ${ }^{2}$ DXP Imaging; ${ }^{3}$ Norton Neuroscience Institute, Norton Healthcare, Louisville, KY; ${ }^{4}$ Department of Neurological Surgery, University of Virginia, Charlottesville, VA; ${ }^{5}$ Department of Neurology, University of Kentucky College of Medicine; ${ }^{6}$ Department of Statistics, University of Kentucky, Lexington; ${ }^{7}$ Doctor Talk, LLC; ${ }^{8}$ Headache Medicine, Norton Neuroscience Institute, Norton Healthcare; ${ }^{9}$ Precision Medicine; ${ }^{10}$ Radiation Oncology, Norton Cancer Institute, Norton Healthcare, Louisville; ${ }^{11}$ Departments of Neurosurgery, Neurology, Radiology, and Neuroscience, University of Kentucky, Lexington, KY, USA

\subsection{6/neurintsurg-2021-ESMINT.55}

Introduction Phase II, single-arm, prospective trial in patients with steroid-refractory brain radiation necrosis (RN).

Aim Evaluate safety and efficacy of single low-dose targeted intra-arterial (IA) bevacizumab following osmotic blood-brain barrier disruption (BBBD).

Methods Following BBBD, 10 adults underwent targeted 2.5 $\mathrm{mg} / \mathrm{kg}$ IA bevacizumab. RN, edema, headache, steroid dependency were quantified at baseline and 12-months. Data (mean \pm SEM) analyzed using Wilcoxon signed-rank tests and oneway repeated measures ANOVA test of linear trend. Null hypothesis rejected for $\mathrm{p}<0.05$.

Results RN decreased by $74.4 \pm 5.2 \%$ with significant linear trend $[\mathrm{F}(1)=10.940, \mathrm{n}=8, \mathrm{p}=0.013$, effect size $=0.610]$. Vasogenic edema decreased by $50.1 \pm 13.2 \%$ but linear trend did not reach significance $[\mathrm{p}=0.102]$. Headache decreased by 84.4 $\pm 6.5 \%$ with significant linear trend $[F(1)=9.299, n=8$, $\mathrm{p}=0.019$, effect size $=0.571]$. Only $1 / 10$ patients were steroid dependent 12-months after bevacizumab. 0/10 died or exhibited AEs attributed to bevacizumab alone. 4 AEs of short duration and moderate severity were probably related to $\mathrm{BBBD}$ (tonic/clonic seizures with altered mental status 2-hours post-procedure) or BBBD+bevacizumab (mono-ocular blurred vision with diplopia on day 1 ). 2/10 patients who experienced marked improvement at 3 months exhibited $\mathrm{RN}$ recurrence requiring intervention (surgery at 10-months; 4-cycles IV bevacizumab at 11-months, respectively).
Conclusions Single low-dose targeted intra-arterial bevacizumab led to durable radiographic and clinical improvement of RN during 12 -months follow-up in $8 / 10$ patients. To our knowledge this is the first prospective report of this novel approach in adults. Randomized trials are needed comparing targeted low-dose IA bevacizumab to multi-cycle IV bevacizumab at higher doses to determine which is longer-lasting, safer, cheaper alternative in brain $\mathrm{RN}$.

\section{REFERENCES}

1. Dashti SR, Spalding A, Kadner RJ, et al. Targeted intraarterial anti-VEGF therapy for medically refractory radiation necrosis in the brain. I Neurosurg Pediatr 2015:15(1):20-25. doi:10.3171/2014.9.PEDS14198

2. Levin VA, Bidaut L, Hou P, et al. Randomized double-blind placebo-controlled trial of bevacizumab therapy for radiation necrosis of the central nervous system. Int $J$ Radiat Oncol Biol Phys 2011;79(5):1487-1495. doi:10.1016/j.jijrobp.2009.12.061

Disclosure Nothing to disclose

\section{EP57 REAL WORLD EXPERIENCE OF THE MIVI QTM ASPIRATION CATHETER}

J Wareham. Department of Interventional Neuroradiology, North Bristol NHS Trust, Bristol, UK

\subsection{6/neurintsurg-2021-ESMINT.56}

Introduction The MIVI $\mathrm{Q}^{\mathrm{TM}}$ Aspiration Catheter offers a range of novel aspiration catheters that have been shown to achieve significantly greater flow rates than other intracranial aspiration catheters in vitro. ${ }^{1}$ Little published data however exists as to how this translates in real world practise.

Aim We describe our initial experience with the MIVI $Q$ in emergent large (TICA,M1,BA) and medium (A1,A2,M2,M3,P1, P2) vessel occlusive stroke.

Methods Data was collated from a prospectively maintained database. Patient demographics, thrombectomy technique, reperfusion scoring and disposition were assessed.

Results Twenty-nine target vessel occlusions occurring in 27 patients were included. Of these 29, 16 (55\%) were large vessel and $13(45 \%)$ medium vessel occlusions. TICI 2C/3 was achieved in 15 (94\%) of large vessel occlusions with 10 $(62.5 \%)$ occurring on first pass, $5(50 \%)$ of which incorporated the simultaneous use of a stent retriever. Successful reperfusion (TICI 2C/3) was achieved in 10 (77\%) medium vessel occlusions with all $10(77 \%)$ occurring on first pass. A stent retriever was used in combination in 1 (10\%). In one patient, an M2 occlusion was withdrawn proximally into the M1. This was removed with aspiration and a stent retriever. No cases of symptomatic intracranial haemorrhage were experienced.

Conclusion The MIVI Q is both safe and effective. Our real world experience supports the superior flow rates demonstrated in vitro and translates into high rates of recanalisation in large and medium vessel occlusive stroke in clinical practise.

\section{REFERENCE}

1. Long TD, Kallmes DF, Hanel $R$, et al. Novel aspiration catheter design for acute stroke thrombectomy. Journal of Neurolnterventional Surgery 2019:11:190-195.

Disclosure Consultant for MIVI Neuroscience 